\title{
Practical Advantages of CBCT in the Surgical Treatment of Impacted Lower Third Molar
}

\begin{abstract}
Alina Ormenișan, Alina lacob*, Daniel Szava, Balint Bogozi, Adina Coșarcă
University of Medicine and Pharmacy Tirgu Mures, Romania

Introduction: The imaging method of cone beam is an improved, extremely accurate computed tomography applicable in the whole field of dentistry. Due to its ability to locate the exact position of the impacted teeth, CBCT software has an important role in the management of difficult cases of impacted third molar. In some situations, the lower third molar is quite near to the inferior alveolar nerve that the surgical extraction can present a high risk of post-operative sensitive impairs of the skin and mucosa of the lower lip and chin on the same side. Presentation of case series: Our study tried to assess the contribution of CBCT in the pre-operative evaluation and further treatment of patients with impacted third molars in mandibular bone with high risk of inferior alveolar nerve injury. The paper presents three clinical cases showing positive signs on standard OPG, which exhibit indicators of a potential contact between the inferior alveolar nerve and the impacted lower third molars. For an improved exploration Dental CT Scan, DICOM image acquisition program, and 3D reconstruction with a special software were used. Conclusions: The study showed that compared with panoramic radiography, CBCT improve the evaluation of the surgical risk and allow a more accurate planning of surgery.
\end{abstract}

Keywords: impacted third molar, CBCT, inferior alveolar nerve, mandibular canal

Received 1 December 2016 / Accepted 16 January 2017

\section{Introduction}

In young adults, over 20 years old, the frequency of maxillary third molars impaction is considered to be about $46 \%$, while the mandibular wisdom teeth impaction is approximately $73 \%$. The incidence is equal for men and women. [1] One of the reasons that third molars inclusion can affect the health of the oral cavity is the large number and the frequency of clinical complications associated with it.[1,2]

The most common complications of the third molar eruption are: infections, dental crowding, tooth decay, periodontal disease, receding gums, loosened teeth, root resorption of adjacent teeth, and difficulties in adapting dental prostheses. Other modifications such as mandibular fractures, development of cysts and tumors, pain in head and neck area, trismus, trophic disorders are much more rare.[3]

To choose the right treatment is necessary to know the position and inclination of the tooth's long axis and the relationship to adjacent structures. Such information can be acquired using radiological examination.[4]

Since the first dental radiography performed in 1896 by Otto Walkhoff [5], radiological investigation methods used in dentistry have developed from standard x-ray images to digital radiology, CT scan, and MRI but especially to CBCT. The expense of MRI and high doses of radiation of classic CT limit their use to selected cases in maxillofacial area.[6]

The emergence of CBCT corrected many deficiencies of existing technology, expanding the use of 3D technology to other fields of dentistry. Cone Beam imaging

* Correspondence to: Alina lacob

E-mail: czmaalina@yahoo.com method named CBCT is based on a perfected computed tomography technology applicable throughout the dentistry area.[7]

In maxillo-facial osseous conditions, CBCT provides information on the precise location of various pathological processes developed in the jaws or facial soft tissues and data about the adjacent anatomical structures.[8]

The presence of certain radiological signs on panoramic radiography such as narrowing, darkening or deflection of the root, dark, bifid or island-shaped apex, interruption of the mandibular canal cortical contour, canal deflection or narrowing, are associated with a veritable relationship between the roots of the third molar and the mandibular canal. However, only cross-sectional CT images obtained by conventional CT or CBCT can define the root-canal relationship in a buccal or lingual direction.[9]

Our study tried to assess the role of CBCT in the treatment of patients with impacted mandibular third molars in a difficult position and high risk of inferior alveolar nerve injury. The injury of the inferior alveolar nerve may represent a rare but serious neurologic complication in the surgery of the impacted third molars requiring a careful pre-operative imagistic evaluation of their anatomical relationship with the inferior alveolar nerve.

\section{Presentation of case series}

Panoramic radiography is considered to be sufficient in most cases prior removing mandibular third molars. However, $\mathrm{CBCT}$ is indicated when one or more signs of close contact between the wisdom tooth and mandibular canal are present in the two-dimensional image.

This paper presents three clinical cases showing positive signs on standard OPG, which exhibit signs of a potential 
contact between the inferior alveolar nerve and the impacted lower third molars. For a better defining the relationship between third molars and the mandibular canal were used Dental CBCT (Cranex 3D provided by Soredex - Tuusula, Finland), DICOM image acquisition program, and $3 \mathrm{D}$ reconstruction with a special software.

\section{Case 1}

A 25-year-old female patient was referred to maxillo-facial surgery department for moderate pain in the right lower jaw. On oral examination, inferior anterior dental crowding and absence of bilateral inferior third molar were identified.

The panoramic radiography (Figure 1) showed mandibular canal image superimposed over the third molar roots, on both sides. Contour lines of the left mandibular canal were barely visible, indicating a most likely lingual location. On the right side was found interrupted the cortical bone of the upper part of the canal and darkening of the roots of the third molar.

To proceed to a safer surgery, the patient underwent a CBCT examination to better evaluate the connection between the mandibular canal and the roots of the third molars (Figure 2). CBCT examination confirmed the lingual position of the left mandibular canal to the roots of left

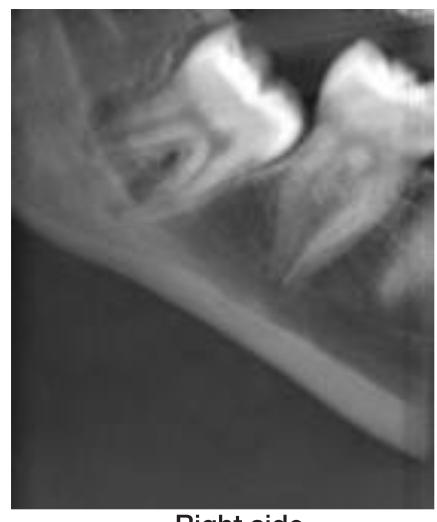

Right side

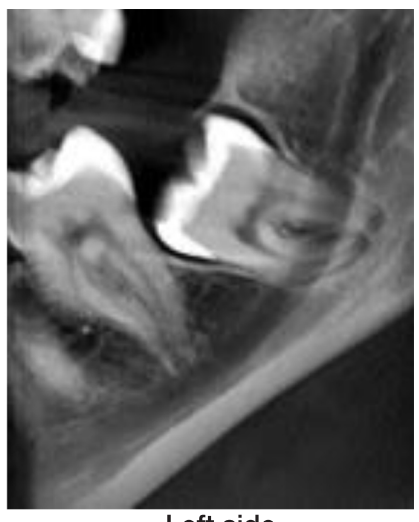

Left side
Fig. 1. Panoramic radiography

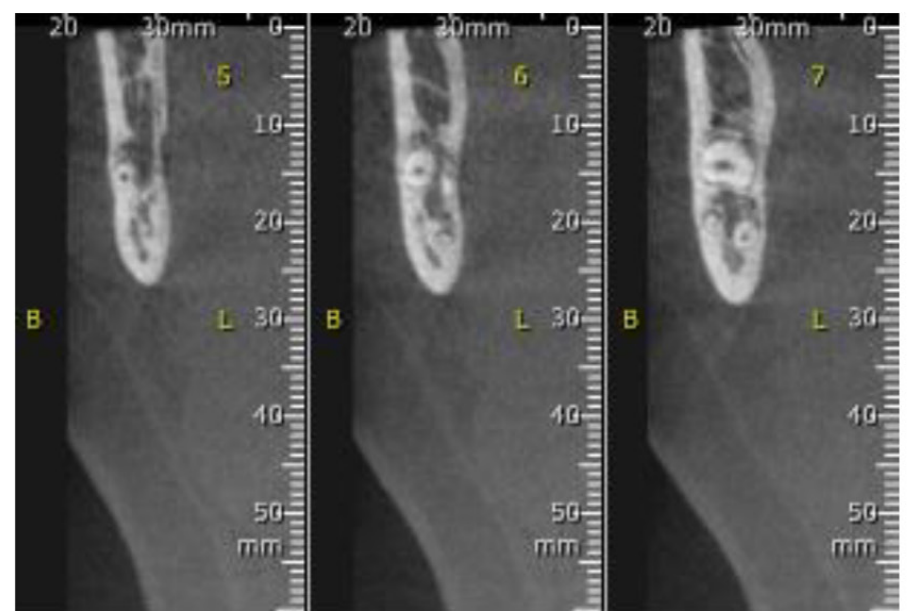

Right side third molar and right mandibular canal crossing through the roots of the right third molar.

The 3-D reconstrucion confirmed the information provided by $\mathrm{CBCT}$ images and offered the possibility to appreciate the arrangement of anatomical details.

After studying CBCT, the surgeon decided to perform odontotomy, roots separation and gentle removing of segments in both third molars in order to avoid inferior alveolar nerve lesion. The patient developed a post-operative sensorial deficiency in left inferior alveolar nerve territory which was recovered almost completely in 8 weeks.

\section{Case 2}

A 22-year-old woman was referred to our department for pain, radiating from the left lower jaw to the left ear which appeared approximately one week ago, with increasing intensity, that did not respond to ibuprofen and metamizole. On intraoral examination, was found left inferior third molar in an abnormal position, with extensive caries and dental crowding both in the lower and upper dental arches.

On panoramic radiography (Figure 3.) was observed partial inclusion of both mandibular third molars with complete root formation. The left third molar had a mesial-angulated position, in contact with the second molar, showing a deep carious process and mesial bone resorp-

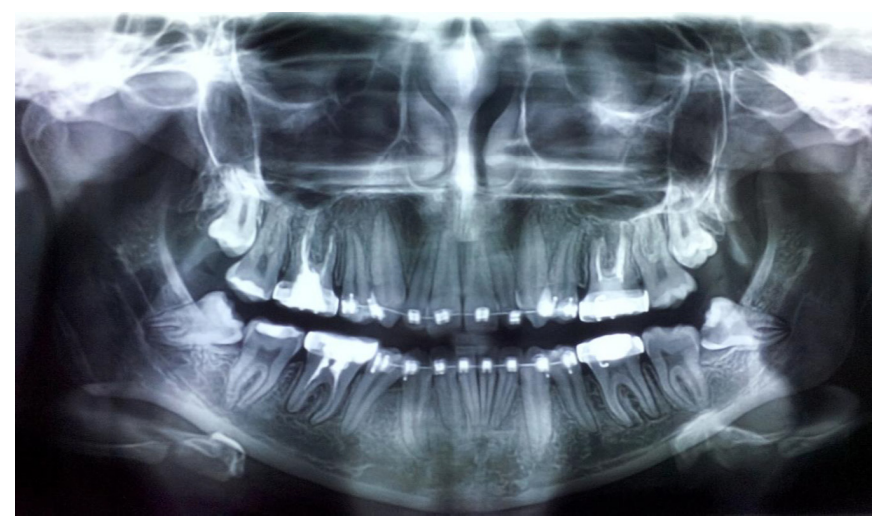

Fig. 3. Panoramic radiography with evident signs of close relationship of both lower third molars with mandibular canal

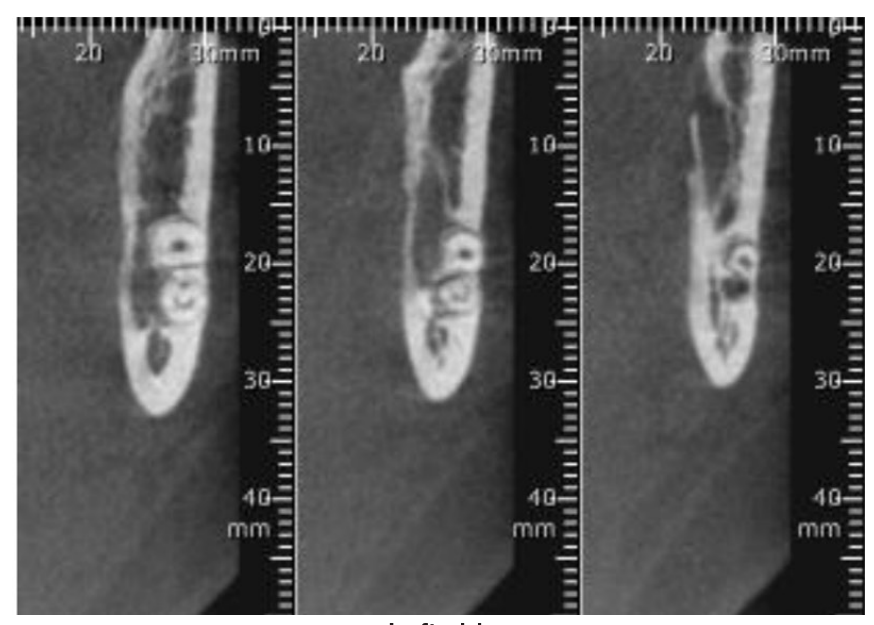

Left side

Fig. 2. СВСТ section showing the intimate relationship between lower third molars roots and mandibular canal 
tion. The roots were convergent overlapping on the mandibular canal. The mandibular canal cortical lines appeared well defined showing a slight deviation near the root apex.

On CBCT examination were specified the position of the left mandibular canal near the vestibular cortical and it contacts with the third molar roots (Figure 4.).

CBCT analysis indicated the position of the roots in contact with the mandibular canal and high risk of inferior alveolar nerve harming, the surgical option being modified for careful separate roots removal. The patient presented slight sensorial deficiency at the left lower lip level which has recovered in 2-3 weeks.

\section{Case 3}

A 24-year-old woman was examined in the emergency service for severe, radiating pain at the right lower jaw, which started 24 hours ago, with no obvious response to ibuprofen. Her medical and dental histories were unremarkable.

On panoramic radiography (Figure 5.) were found both mandibular third molars partially impacted with complete root formation. At the right second molar was observed a distal cavity associated with impacted third molar in a mesial incline. The right third molar presented roots overlapping on the mandibular canal whose shape appears as two radiopaque, net lines, which can be easily distinguished, not showing deviations, probably in a vestibular position.

On CBCT examination was identified a vestibular position of the mandibular canal and a punctiform contact with the roots of the right third molar (Figure 6.).

Surgical plan was modified from initial odontectomy to odontotomy with roots separation. The patient presented no post-operative sensory impairment.

\section{Discussion}

In oral and maxillo-facial surgery, panoramic radiography is the first-level imaging of choice in the pre-operative evaluation of the third molar.[10]

Radiographic signs, detectable on the panoramic radiography that indicate the presence of a close relationship between the inferior alveolar nerve and the lower third molar are [11,12]:

- Radiotrasparent band darkening the root of the third molar because of the decreasing of bone density produced by the mandibular canal crossing the area.

- Interruption of the line marking the roof of the canal due to the root of the third molar crossing it.

- Sudden change of direction or narrowing of the mandibular canal at the point in which it is in contact with or superposed on the roots of the third molar.

- Abrupt deviation of the roots of the third molar at the point where they superpose on or come in contact with the mandibular canal.

- Bifid apex with third molar root darkening or root depression at the point where they are crossed by the inferior alveolar nerve.

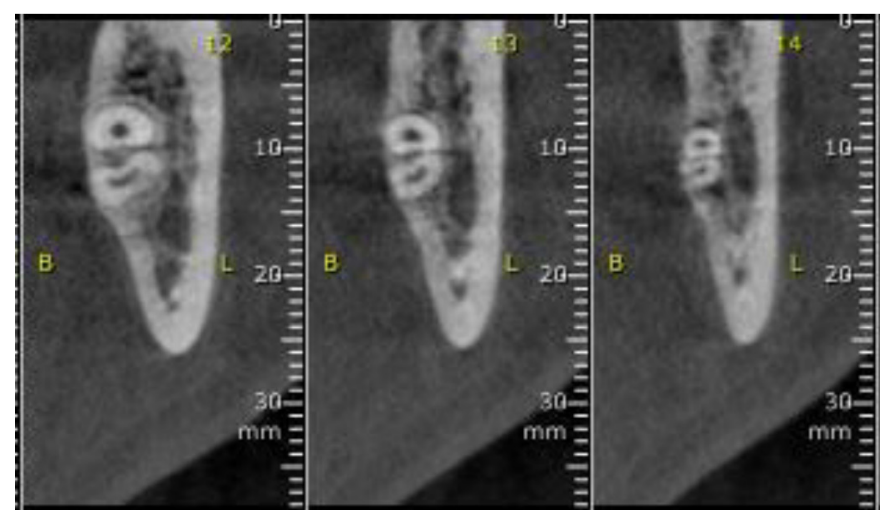

Fig. 4. CBCT sections indicating the left lower third molar roots in contact with mandibular canal

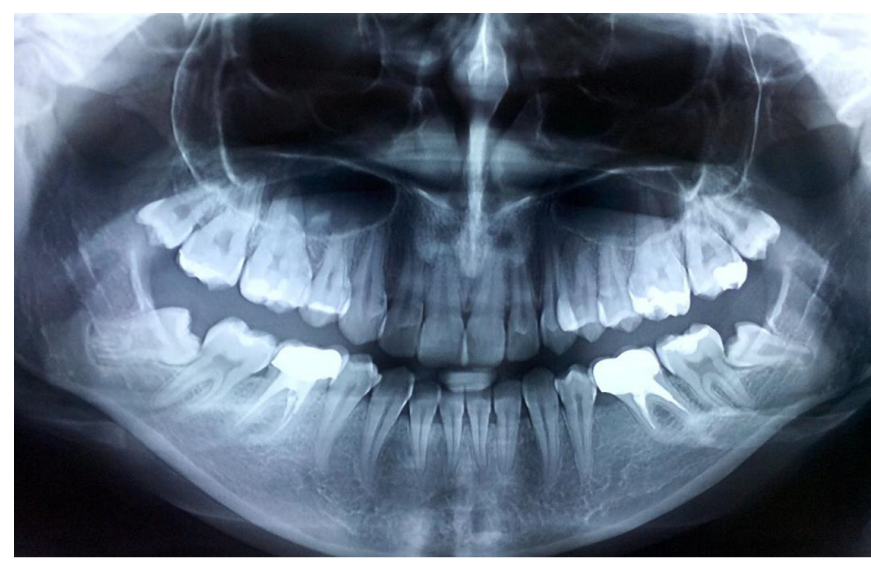

Fig. 5. Panoramic radiography that indicate signs of right trird molar roots in posible contact with mandibular canal

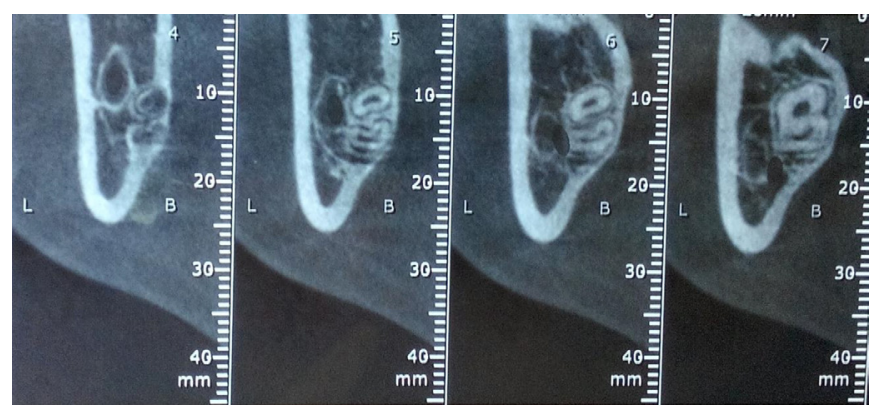

Fig. 6. СВСТ sections showing the contact of the right lower third molar roots with mandibular canal

- Superposition of the roots of the third molar and the mandibular canal.

- The roots of the third molar are in contact with the roof of the mandibular canal

There are studies which report that darkening of the roots, interruption of the cortex of the roof of the canal, and canal deviation detected pre-operatively are the radiographic signs that are most often associated with inferior alveolar nerve exposure and intraoperative injury. $[10,13,14,15]$

The absence of positive radiographic signs on the panoramic radiography is preferable to their presence for preoperative diagnostic purposes and for a reliable surgical approach. Without positive radiographic signs on pano- 
ramic radiography, the risk of inferior alveolar nerve injury is considered to be small, but the presence of one or more signs might indicate a high probability of intra-operative exposure of the vascular-nervous bundle.[16]

Being a bi-dimensional examination, OPT does not provide information on the depth of the anatomical structures studied and locates the mandibular canal only in the vertical and not in the horizontal plane. On the other hand, it provides a distorted magnification by a variable factor greater horizontally than vertical, and anatomical structures are overlapped - air shadows, soft tissues, and phantom images. [16,17]

Some authors compared the diagnostic accuracy of OPT vs. CBCT in detecting the relationship between the root apex of third molars and the mandibular canal. A significant difference was found between the two techniques on the horizontal plane, but the diagnostic information provided by CBCT was far better.[18]

Most authors consent that CBCT and Dental CT Scan are the most effective imaging techniques to identify the localization of the mandibular canal regarding its superior/ inferior and buccal/lingual dimensions, and the precise crown-root morphology of third molars.[18]

CT examination should be used only for patients in whom panoramic radiography shows one or more of the radiographic signs indicating an intimate relationship between the mandibular canal and the third molar root, but this relationship cannot be defined sufficiently using conventional radiology. [12,15,19,20] Thereby, it may substantially contribute on the planning of the surgical approach and on the evaluation of consequences and results. [16]

CBCT gives undistorted three-dimensional images with a very good resolution that allows visualization of anatomical structures shape and their real size.[21]

CBCT exam permits to evaluate the buccolingual relationship between the mandibular canal and the roots of the third molar thus avoiding to push the tooth during surgical movements and to hurt the inferior alveolar nerve. In this way can be planned the appropriate interradicular section if it is evident that the inferior alveolar nerve crosses the roots. CBCT exam can also identify the presence or the absence of cortical bone around the inferior alveolar nerve and allows to detect the number of roots of the third molar and the precise their anatomy. Furthermore, $\mathrm{CBCT}$ determines the inclination of the tooth and the position of the crown in relation to the buccal or lingual surface of the mandible.[16,22]

The usage of CBCT has reduced the cost for patients, and mostly it has improved the risk-benefit ratio by reducing the dose of radiations for patients compared to standard CT exam.[15] In cases where the roots of the third molars have a complex morphology being located in contact with the mandibular canal, the new $3 \mathrm{D}$ reconstruction programs assisting CT images provide sharp visualization in the three spatial planes of the structures from the mandibular canal that have to be respected.[16]

$3 \mathrm{D}$ images are not mandatory for the pre-operative evaluation of third molars. They only complete anatomical image's details that might influence the surgical approach: single or multiple odontotomy, depth of osteotomy or direction of luxation which can be programmed more accurately.[16]

\section{Conclusions}

CBCT is an excellent diagnostic method for selected situations in oral and maxillo-facial surgery, including evaluation of mandibular third molars, but its efficiency has been lesser studied yet. Panoramic radiography may be sufficient in most cases before removal of mandibular third molars, but CBCT may be indicated when one or more signs of close contact between the tooth and the mandibular canal are present in the standard panoramic radiography. In these situations, $\mathrm{CBCT}$ might change the surgical approach and patient's outcomes.

The study have shown that CBCT contributes to optimal risk assessment and an adequate surgical planning, compared with panoramic radiography.

The mandibular morphology at the third molar region with impacted teeth and the location of the mandibular canal could be distinctly determined using cross-sectional CBCT images.

\section{Acknowledgment}

This paper was partially sustained by the Project No. 912/2015 financed by S.C. OPTOMED SRL in collaboration with UMF TG. MURES.

\section{Conflict of interest}

None to declare.

\section{References}

1. Carter K, Worthington S. Predictors of Third Molar Impaction: A Systematic Review and Meta-analysis. J Dent Res. 2016 Mar;95(3):26776.

2. Huang GJ, Cunha-Cruz J, Rothen M, Spiekerman C, Drangsholt M, Anderson L, Roset GA. A Prospective Study of Clinical Outcomes Related to Third Molar Removal or Retention. Am J Public Health. 2014;104(4):728-234.

3. American Association of Oral and Maxillofacial Surgeons, The Management of Impacted Third Molar Teeth, 2013

4. Chandak S, Shetty CM - Comparative Study of Dentascan and Radiography for Radiological Evaluation of Impacted Teeth, J Clin Diagn Res, 2014, 8(7): RC01-RC05.

5. Sansare K, Khanna V, Karjodkar - Early Victims of X-rays: a Tribute and Current Perception, Dentomaxillofac Radiol, 2011, 40(2): 123-125.

6. Miles DA - Metoda Imagistică cu Fascicul Conic - "De ce Atâta Agitație în Legătură cu Doza de Radiații?", Revista de Imagistică Dentară, 2010, 2: 14-22.

7. Matzen LH, Wenzel A - Efficacy of CBCT for Assessment of Impacted Mandibular Third Molars: a Review - Based on a Hierarchical model of evidence, Dentomaxillofac Radiol, 2015, 44(1): 20140189.

8. Gonzalez SM - Interpretation Basics of Cone Beam Computed Tomography, Wiley Blackwell, lowa, 2014, 16-22.

9. de Melo Albert DG, Gomes AC, do Egito Vasconcelos BC, de Oliveira e Silva ED, Holanda GZ. Comparison of orthopantomographs and conventional tomography images for assessing the relationship between 
impacted lower third molars and the mandibular canal. J Oral Maxillofac Surg. 2006;64:1030-37.

10. Atieh MA. Diagnostic accuracy of panoramic radiography in determining relationship between inferior alveolar nerve and mandibular third molar. J Oral Maxillofac Surg. 2010;68:74-82.

11. Koong B, Pharoah MJ, Bulsara M, Tennant M. Methods of determining the relationship of the mandibular canal and third molars: a survey of Australian oral maxillofacial surgeons. Aust Dent J. 2006;51:64-68.

12. Friedland B, Donoff B, Dodson TB. The use of 3-dimensional reconstruction to evaluate the anatomic relationship of the mandibular canal and impacted mandibular third molars. J Oral Maxillofac Surg. 2008;66:1678-1685.

13. Dodson TB. Role of computerized tomography in management of impacted mandibular third molars. N Y State Dent J. 2005 Nov

14. Bell GW. Use of dental panoramic tomographs to predict the relation between mandibular third molar teeth and the inferior alveolar nerve. Radiological and surgical findings, and clinical outcome. Brit J Oral Maxillofac Surg. 2004;42:21-27

15. Sedaghatfar M, August MA, Dodson TB. Panoramic radiographic findings as predictors of inferior alveolar nerve exposure following third molar extraction. J Oral Maxillofac Surg. 2005;63:3-7.
16. Tuzi A, Di Bari R, Cicconetti A. 3D imaging reconstruction and impacted third molars: case reports. Ann Stomatol (Roma). 2012 Jul-Dec; 3(3-4): 123-131.

17. Liu T, Xia B, Gu Z. Inferior alveolar canal course: a radiographic study. Clin Oral Implants Res. 2009;20:1212-1218

18. Neugebauer J, Shirani R, Mischkowski RA, Ritter L, Scheer M, Keeve E, Zoller JE. Comparison of cone beam volumetric imaging and combined plain radiographs for localization of the mandibular canal before removal of impacted lower third molars. Oral Surg Oral Med Oral Pathol Oral Radiol End. 2008;105:633-42.

19. Flygare L, Ohman A. Preoperative imaging procedures for lower wisdom teeth removal. Review. Clin Oral Invest. 2008;12:291-302

20. Susarla SM, Dodson TB. Preoperative computed tomography imaging in the management of impacted mandibular third molars. J Ora Maxillofac Surg. 2007;65:83-88.

21. Suomalainen A, Esmaeili EP, Robinson S - Dentomaxillofacial Imaging with Panoramic Views and Cone Beam CT, Insights Imaging, 2015, 6(1): 1-16.

22. Pippi R. A case of inferior alveolar entrapment in the roots of a partially erupted mandibular third molar. J Oral Maxillofac Surg. 2010;68:11701173 